EPJ Web of Conferences 92,02076 (2015)

DOI: $10.1051 /$ epjconf/ 20159202076

(C) Owned by the authors, published by EDP Sciences, 2015

\title{
Numerical simulation of deformation of dynamic mesh in the human vocal tract model
}

\author{
Václav Řidký, ${ }^{1 a}$ and Petr Šidlof ${ }^{2}$ \\ ${ }^{1}$ Institute of Thermomechanics AS CR, v. v. i., Dolejškova 1402/5, 18200 Praha 8, Czech Republic
}

\begin{abstract}
Numerical simulation of the acoustic signal generation in the human vocal tract is a very complex problem. The computational mesh is not static; it is deformed due to vibration of vocal folds. Movement of vocal folds is in this case prescribed as function of translation and rotation. A new boundary condition for the 2DOF motion of the vocal folds was implemented in OpenFOAM, an open-source software package based on finite volume method Work is focused on the dynamic mesh and deformation of structured meshes in the computation a package OpenFOAM. These methods are compared with focus onquality of the mesh (non-orthogonality, aspect ratio and skewness).
\end{abstract}

\section{Introduction}

In this time exists many physical problems where you do not make simulation on static mesh. Big group of physical phenomena are solid-fluid interactions. Where prescribed motion on the boundary surface make change shape of computational domain. Typical case of fluid-solid interaction is generation of human voice. A result of this interaction is oscillation of the human vocal folds in time. This paper is focused on issue of dynamic mesh in computing package OpenFOAM.

In the deforming mesh method, the computational mesh is updated every time step. In the paper are present simulations, the motion is prescribed on boundary layers. Internal points of the computational domain are translating and rotating. During this process is very important to check mesh quality after the time step. If the shape of the domain boundary is time-varying it is important that the internal mesh preserves it is validity (no negative cell volume), quality, and boundary closure. In computational package OpenFOAM are main parameters of the quality of the finite volume mesh. These parameters are non-orthogonality, aspect ratio and skewness.

For numerical solution of the deformation several approaches can be used. One of these approaches is spring analogy [3]. Here, all edges between the points of mesh are replaced by linear springs. Next method is Radial basic function (RBF). This method is for the motion of the human vocal inconvenient. In the OpenFOAM used to Radial basic function for computing deformation mesh pseudo-solid method [2].

\footnotetext{
${ }^{\mathrm{a}}$ Corresponding author: vaclav.ridky@tul.cz
}

The next chapter describes in more detail the techniques for solution of the mesh deformation in OpenFOAM. These techniques are compared and results are shown for the case of the human vocal tract.

\section{Approaches for calculation of the mesh deformation in OpenFOAM}

\subsection{Algebraic Mesh Motion}

Algebraic mesh motion solver using for position and velocity of points of mesh directly calculated from a globally known motion laws. The motion technique is exceptionally efficient and accurate but somewhat limited in scope.

\subsection{Laplacian}

Another approach is to use the Laplace, which is a variant of the linear stress equation [5]. A mesh motion method based on one of these equations is computationally cheap since the resulting matrix system is sparse, such that existing iterative solvers can be used efficiently.

The nature of the Laplace equation is that the point displacements will be largest close to the moving boundary and small at large distance. Ideally, a user input is not desired, since it decreases the robustness of the method. However, this method needs the specification of a variable diffusivity. This leads to the following definition of the Laplace equation:

$$
\nabla \cdot(r \nabla u)=0
$$


Where $\boldsymbol{r}$ is constant variable diffusion coefficient and $\boldsymbol{u}$ is the velocity of displacement. Diffusion depends on the distance from the moving boundary. This dependence is described by the function:

$$
r(\mathrm{r})=1 / \mathrm{r}^{\mathrm{m}}
$$

The resulting mesh quality strongly depends on the chosen $\gamma(\mathrm{r})$ function, which depends on the distance from the moving boundary. This variable diffusion coefficient can be chosen such that a region next to the deforming or moving boundary closely moves with the boundary. The resulting mesh contains less cell quality deterioration next to the boundary. The current research uses a $\gamma(\mathrm{r})$ function like equation (2). In addition to the freedom of choosing a diffusion function, it is also possible to define $\gamma(\mathrm{r})$ for every internal mesh cell for all time-steps independently. This, however, appears to be very problem dependent and thus optimization of $\gamma(\mathrm{r})$ seems not cost effective. To maintain robustness, in the current work we use a quadratic, $\mathrm{m}=2$, decreasing diffusion coefficient, which was found to provide efficient and a smooth mesh motion [1]. Additionally, one could also have used an exponentially decreasing diffusion coefficient or a diffusion coefficient related to the mesh deformation energy.

\subsection{Pseudo-solid}

For cases where boundary motion is irregular or solutiondependent, algebraic mesh motion is not sufficiently flexible. [4] An alternative way of looking at the mesh motion problem is to consider prescribed boundary motion as a boundary condition on an unknown mesh motion equation. It follows that internal point motion may be determined by solving the motion equation. The solution of deformation describes equation for small deformation:

$$
\nabla \cdot\left[r\left(\nabla \mathbf{u}+(\boldsymbol{V} \nabla \mathbf{u})^{T}\right)+r[\nabla \cdot \mathbf{u}]=0\right.
$$

Variables are described in the previous chapter.

\subsection{Mesquite}

Mesquite is algorithm for approach, which is based on control of the mesh quality. A global norm of the mesh quality is calculated and optimization performed. For the computation of the deformation of the mesh is used the pseudo-solid algorithm.

\subsection{Radial Basic Function interpolations}

Radial basis function (RBF) interpolation is based on finding of the displacement of the internal points for a given boundary displacement. The interpolation is described by equation:

$$
s(x) \sum_{i=1}^{N_{i}} y_{j} \phi\left(\left|x-x_{i, j}\right|\right)+q(x)
$$

where $\boldsymbol{x}$ is the interpolated location, $\boldsymbol{x}_{\boldsymbol{b}}$ is the set of $\mathrm{N}_{\mathrm{b}}$ location carrying the data, $\phi(x)$ is the basis function, dependent on point distance and $q(\boldsymbol{x})$ is the polynomial function, depending on choice of the basis function and $\gamma_{j}$ is diffusion coefficient [6]. Consistency of the interpolation is achieved by requiring that all polynomials of the order lower than $q$ disappear at data points

$$
\sum_{j=1}^{N_{n}} x_{j} p\left(x_{i, j}\right)=0
$$

Coefficients $q$ and $\gamma_{\mathrm{j}}$ are solution of system:

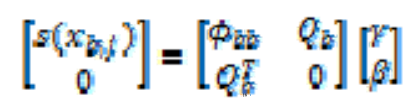

Here is $s\left(\boldsymbol{x}_{b, j}\right)$ the function value at interpolated location, $\phi_{b b}$ carriers the evaluation of the basis function for pairs of interpolation points, $Q_{b}$ is the rectangular matrix and $\gamma$ carriers all $\gamma_{\mathrm{j}}$ coefficients and $\beta$ carries on coefficient of.

\section{Geometry and boundary condition}

\subsection{Geometry}

Geometry of the problem is simple model of human vocal tract. Real human vocal tract is more complicated. This is the area inside the human vocal tract, where is the flow. In this geometry are moving only vocal folds, other walls are static.

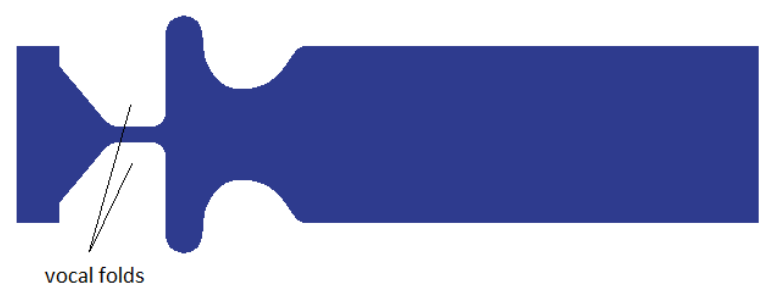

Figure 1. Simple geometry model of human vocal tract.

\subsection{Boundary condition}

For the dynamic mesh is needed in the OpenFOAM prescribed boundary condition for motion. For static walls is prescribed boundary condition with zero displacement. For motion of the vocal folds is implement new boundary condition (user boundary condition). This boundary condition combines translation and rotation motion. The motion is described by harmonic equation

$$
\begin{aligned}
& x_{1}=\mathrm{x}_{10}+\mathrm{X}_{1} * \sin \left(2 \pi \mathrm{f}_{1} * \mathrm{t}+\mathrm{x}_{1 \text { phase }}\right) \\
& x_{2}=\mathrm{x}_{20}+\mathrm{X}_{2} * \sin \left(2 \pi \mathrm{f}_{2} * \mathrm{t}+\mathrm{x}_{2 \text { phase }}\right),
\end{aligned}
$$

where the variable with index one corresponds to translation motion and the variable with index two 
correspond to rotation motion. $\mathrm{x}_{\mathrm{i} 0}$ is the initial value, $\mathrm{X}_{\mathrm{i}}$ amplitude, $f_{i}$ frequency, $t$ time and $x_{\text {iphase }}$ the phase shift.

Because walls with the exception of vocal folds are static is part of implement boundary condition mechanism that monitors the distribution of elements between the vocal folds and neighboring walls. This mechanism controls the deformation of the elements and theirs appropriate distribution in this area. Mesh is generated in a mesh generation tool snappyHexMesh (Figure 1 and Figure 2). Parameters the mesh: structured $2 \mathrm{D}$ (one layer hexahedron), number of elements is $46 \mathrm{k}$.

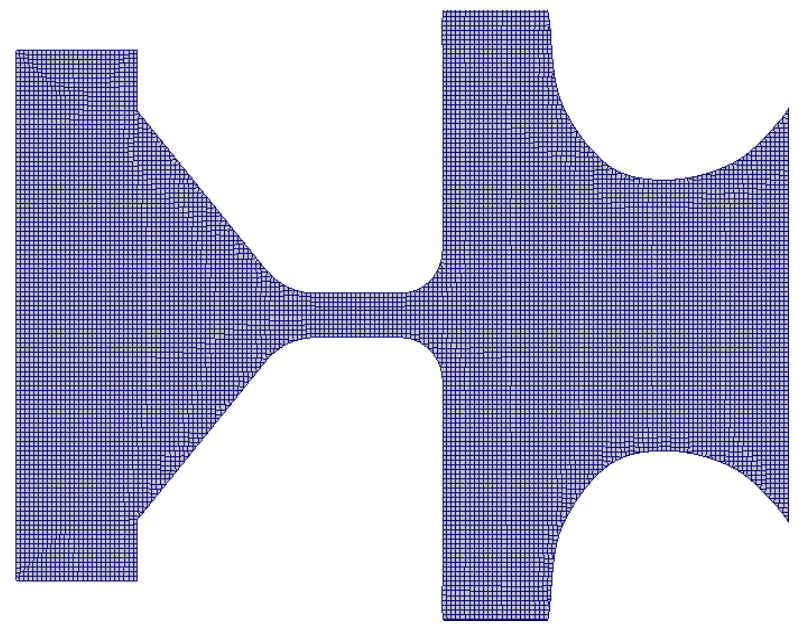

Figure 1. Undeformed mesh of the vocal fold area in time $\mathrm{t}=0 \mathrm{~s}$.

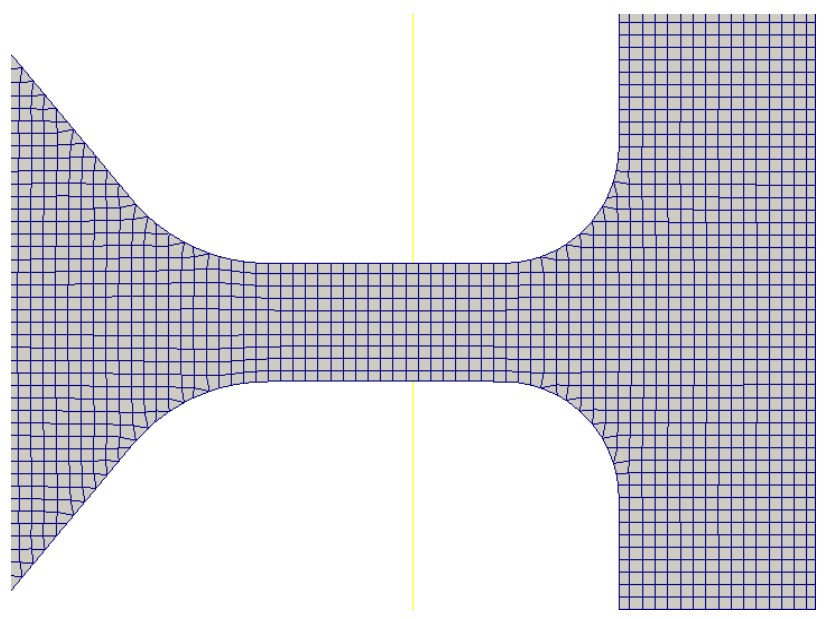

Figure 2. Detail of the mesh between vocal folds in time $t=0$ s.

\section{Results}

The result of the work is evaluation of results of mesh deformation and comparison of various approaches of mesh motion solver. In this case we compare Laplacian, Mesquite and Pseudo-solid approaches. Evaluation of mesh quality is done according to several main parameters. These parameters are non-orthogonality, aspect ratio and skewness. For comparison are values in the Table 1. Computing time corresponds to one period of the harmonic motion (10 ms simulation time).
Table 1. Mesh quality for individual methods in time $t=0.01 \mathrm{~s}$ and mesh quality in time $\mathrm{t}=0 \mathrm{~s}$.

\begin{tabular}{|c|c|c|c|c|}
\hline & Laplacian & Mesquite & $\begin{array}{c}\text { Pseudo- } \\
\text { solid }\end{array}$ & $\begin{array}{c}\text { Mesh in } \\
\text { time } \mathrm{t}=0 \mathrm{~s}\end{array}$ \\
\hline $\begin{array}{c}\text { Average } \\
\text { non- } \\
\text { orthogon } \\
\text { ality }\end{array}$ & 180 & 43,66 & 72,7 & 28,2 \\
\hline $\begin{array}{c}\text { Max } \\
\text { non- } \\
\text { orthogon } \\
\text { ality }\end{array}$ & 37,2 & 2,08 & 2,78 & 0,86 \\
\hline $\begin{array}{c}\text { aspect } \\
\text { ratio }\end{array}$ & $1,8 * 10^{221}$ & 72,87 & 74,29 & 37,4 \\
\hline skewness & 8,21 & 1,49 & 1,4 & 0,82 \\
\hline $\begin{array}{c}\text { Computi } \\
\text { ng time }\end{array}$ & $57 \mathrm{~s}$ & $75 \mathrm{~s}$ & $127 \mathrm{~s}$ & \\
\hline
\end{tabular}

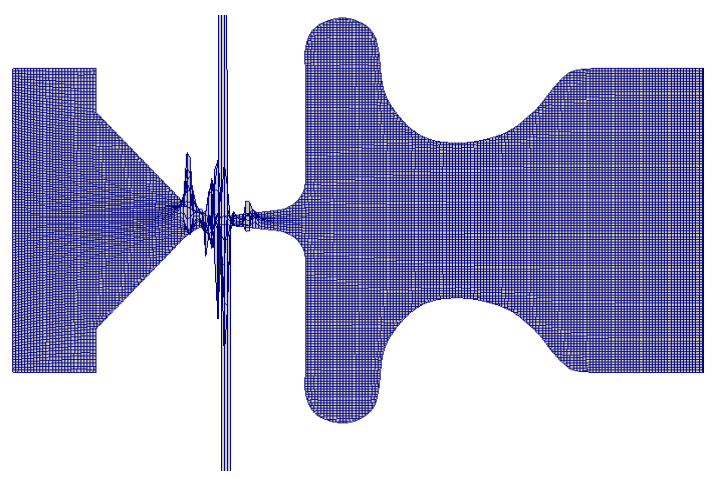

Figure 3. Laplacian method mesh in the time $\mathrm{t}=0.5 \mathrm{~ms}$ (2 time steps).

On the beginning the mesh has the best mesh quality parameter. During deformation of the mesh parameters worsen. From this perspective, the worst is the Laplacian method, because the solution after a few steps completely failed (Figure 3.). With increasing distance from the wall elements are more deformed. In this case also tested and different diffusion coefficients, but the results were similar in all cases, after a few time steps, the mesh was unusable. The parameters are so high that the mesh is unusable for calculations.

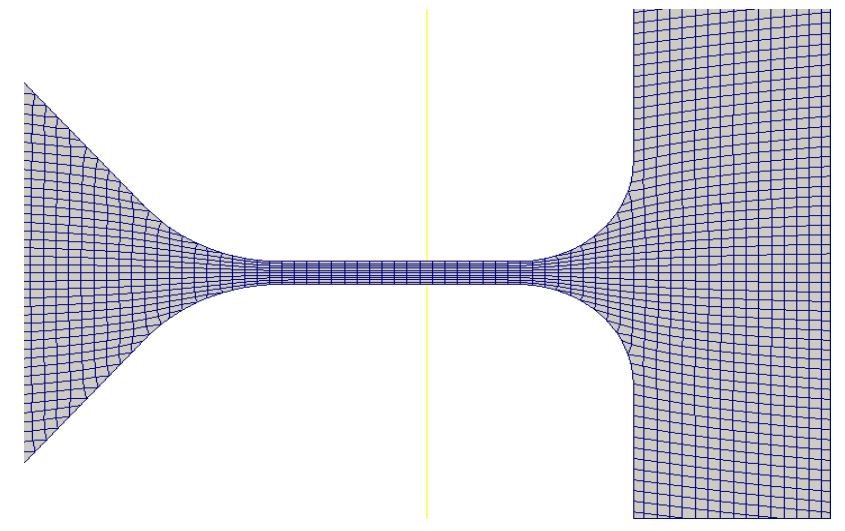

Figure 4. Pseudo-solid method mesh in the time $\mathrm{t}=7 \mathrm{~ms}$. 
Better results of deformation have approaches based on the Pseudo-solid method. Both methods give comparable results. The difference between the methods is due to the failure by Pseudo-solid methods to fully implement the boundary conditions. Broken mechanism, which performs uniform distribution of elements on boundaries (vocal fold and the channel wall), does not work here and so are elements in these parts wrong deformed (Figure 5). In the rest of the field are identical results of deformation since both methods have the same base (Figure 4. and Figure 7.)

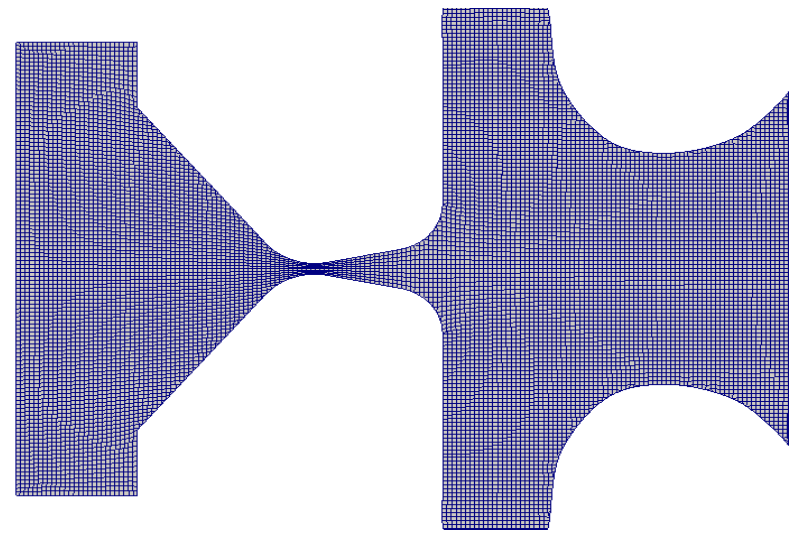

Figure 5. Detail of the mesh in the time $10 \mathrm{~ms}$.

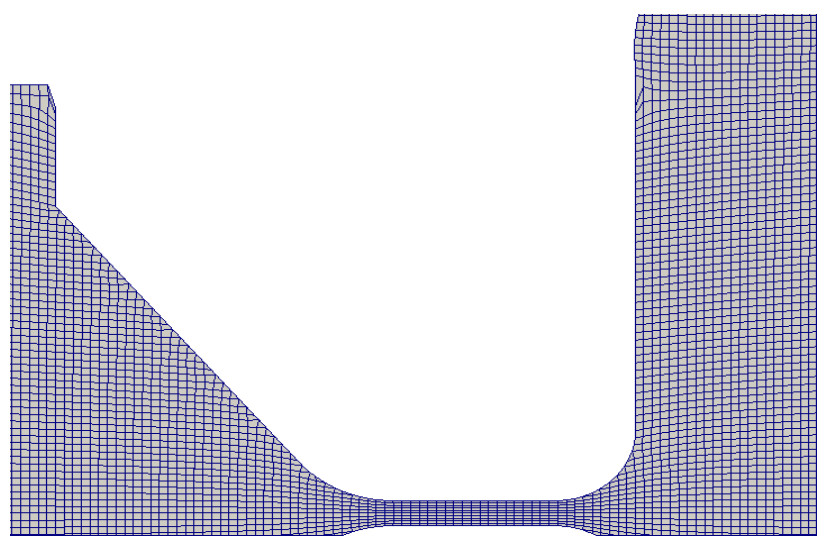

Figure 6. Pseudo-solid method detail wrong mesh deformation between (vocal folds and channel wall).

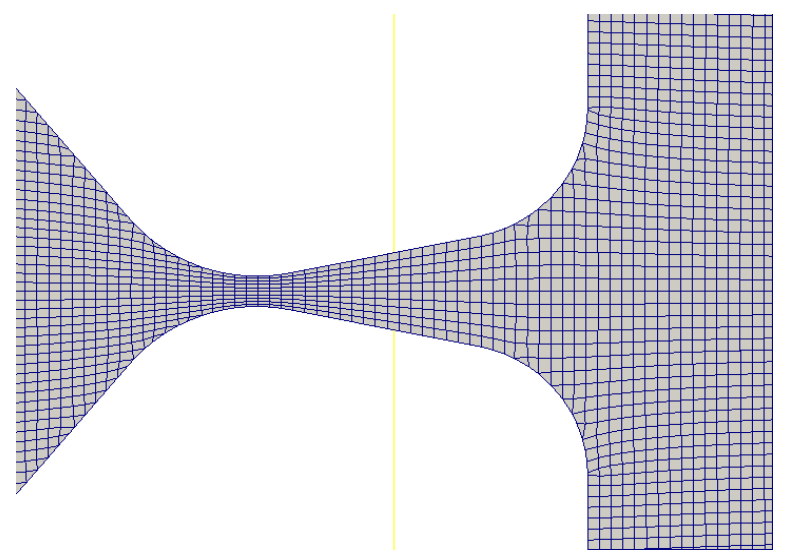

Figure 7. Mesquite method detail of the mesh in the time $\mathrm{t}=10 \mathrm{~ms}$.

\section{Conlusion}

Computational package OpenFOAM offers an extensive variety of approaches for solving deformation mesh. Those are commonly used and are for some types of tasks appropriate. If it is use a boundary condition with prescribing move on to more complex geometries. Where there is a movement towards each two boundaries are simple methods for the calculation of deformation insufficient. Therefore, over time the use methods based on the calculation of small deformations of solids. Another option is to remesh the computational domain before each time step and perform interpolation. This method is very time consuming. Methods based on the calculation of small deformations of solids give very good results even with large narrow the gap between the vocal folds. This is important for flow simulation because here the flow separation occurs.

\section{Acknowledgments}

The research has been supported by the Czech Science Foundation, project P101/11/0207 "Coupled problems of fluid and solid mechanics - nonlinear aeroelasticity".

\section{References}

1. H. Jasak, Z. Tukovic, Transaction of FEMENA 30 (2007)

2. C. Kassiotis, European Journal of Mechanics 11 (2004)

3. F. Blm, Int. J. Num Meth. Fluids 32 (2000)

4. B. Frank, Numerical simulation of flapping foil and wind aerodynamics: Mesh reformatting using radial basic function, ( $\mathrm{PhD}$ Thesis, Technical university Delft 2009)

5. R.P Dwight, Computational Fluid Dynamics (2006)

6. Boer de, A., van der Schoot, H. Bijl, Computers \& Structures 85 (2007) 